\title{
Measurement of global betatron coupling with skew quadrupole modulation
}

\author{
Y. Luo, * P. Cameron, A. Della Penna, Jr., J. Laster, R. Lee, A. Marusic, F. Pilat, T. Roser, D. Trbojevic, and J. Wei \\ Brookhaven National Laboratory, Upton, New York 11973, USA
}

(Received 28 October 2004; published 11 January 2005)

\begin{abstract}
Measurement of the residual betatron coupling with skew quadrupole modulation is a new diagnostics technique. It was developed and tested at the Relativistic Heavy Ion Collider (RHIC) as a promising method for measuring coupling on the ramp. By modulating the strengths of skew quadrupole families, the two tunes' responses are precisely measured with the phase lock loop system. The projection ratio of the residual coupling coefficient onto the coupling modulation direction can be determined. In this article, the analytical solution to the skew quadrupole modulation is given. Dedicated beam studies were carried out in RHIC Run'04 and the results are presented. The ability to measure coupling on the ramp opens the possibility of continuously correcting coupling during acceleration.
\end{abstract}

DOI: 10.1103/PhysRevSTAB.8.014001

PACS numbers: 29.20.Dh, 29.27.Bd

\section{INTRODUCTION}

For a hadron machine like the Relativistic Heavy Ion Collider (RHIC), the working points are constrained into a very narrow space. Thus, the two tunes must be kept close to the linear difference resonance line to ensure longer beam lifetime. During the RHIC's polarized proton operations, the betatron coupling should be especially well corrected on the ramp to avoid crossing of the spin tune resonances and loss of polarization. Coupling correcting also helps the vertical closed orbit correcting, which benefits the polarization's preservation.

Skew quadrupole strength scan is the conventional method to measure and correct the global coupling [1-4]. However, it is not suitable for the coupling measurement on the ramp because of its several disadvantages [5]. Before the skew quadrupole scan, the two tunes should be pushed to the linear difference coupling resonance. Then the skew quadrupole strengths are scanned to search the minimum tune split. During the scan, large skew quadrupole strength may lead to beam loss. And close to the minimum tune split, the tune changes are very small, so it is not easy to determine where the minimum tune split really is. After the scan, the tunes should be restored to their original values.

As a logical extension, we proposed the skew quadrupole modulation technique to replace the skew quadrupole scan. The tune split is still used as the observable during the modulation. The analytical solution to the tune split during the modulation was found, based on the Hamiltonian perturbation theory of linear difference coupling. In order to track the fast tune changes, the phase lock loop (PLL) system $[6,7]$ is adapted. Different data processing methods were found to extract the residual coupling's projection ratio onto the coupling modulation direction. This technique has several advantages compared to the skew quadrupole scan. First, it is not necessary to move the tunes to

*Electronic address: yluo@bnl.gov the linear difference coupling resonance before the modulation. Second, the skew quadrupole modulation is fast and safe, which makes it possible for the global coupling measurement on the ramp. The period occupied by one projection measurement is reduced to several seconds.

In this article we first give the analytical solution to the tune split from the skew quadrupole modulation. Then, different data processing methods to get the projection ratio are presented. The preliminary experimental results at RHIC are discussed.

\section{SKEW QUADRUPOLE MODULATION}

\section{A. Perturbation theory}

From linear difference coupling's Hamiltonian perturbation theory $[1,2,8]$, the two tunes $Q_{1,2}$ in the coupled situation are given by

$$
\begin{aligned}
& Q_{1}=Q_{x, 0}-\frac{\Delta}{2}+\frac{1}{2} \sqrt{\Delta^{2}+\left|C^{-}\right|^{2}}, \\
& Q_{2}=Q_{y, 0}+\frac{\Delta}{2}-\frac{1}{2} \sqrt{\Delta^{2}+\left|C^{-}\right|^{2}},
\end{aligned}
$$

where $Q_{x, 0}, Q_{y, 0}$ are the uncoupled tunes when all coupling sources are removed. $\Delta$ is the tune split of the two uncoupled tunes,

$$
\Delta=Q_{x, 0}-Q_{y, 0}-p
$$

where $p$ is the integer tune split. $C^{-}$is the coupling coefficient, which normally is a complex number. It is defined as

$$
C^{-}=\left|C^{-}\right| e^{i \chi}=\frac{1}{2 \pi} \oint \sqrt{\beta_{x} \beta_{y}} k_{s} e^{i\left[\Phi_{x}-\Phi_{y}-\Delta(2 \pi s / L)\right]} d l,
$$

where $\left|C^{-}\right|$is coupling amplitude, $\chi$ is the angle of the coupling, $\beta_{x}$ and $\beta_{y}$ are the linear horizontal and vertical betatron amplitude functions, $\Phi_{x}$ and $\Phi_{y}$ are the betatron phase advances, $k_{s}$ is the skew quadrupole strength, $L$ is the 
ring circumference, and $s$ is the distance between the skew quadrupole location and the reference point to calculate the coupling.

\section{B. Tune split from modulation}

From Eq. (1), the tune split $|\Delta Q|$ is given by

$$
|\Delta Q|=\left|Q_{1}-Q_{2}-p\right|=\sqrt{\Delta^{2}+\left|C^{-}\right|^{2}} .
$$

This tune split $|\Delta Q|$ can be measured experimentally.

If one skew quadrupole's strength modulates, from Eq. (3), its coupling contribution to the total coupling in the ring also modulates,

$$
C_{\text {mod }}^{-}=C_{\text {mod,amp }}^{-} \sin (2 \pi f t),
$$

where $C_{\bmod }^{-}$is the coupling introduced by the skew quadrupole modulation, $C_{\text {mod,amp }}^{-}$is its amplitude, and $f$ is the modulation frequency.

The total coupling $C_{\text {tot }}^{-}$in the ring is

$$
C_{\text {tot }}^{-}=C_{\text {res }}^{-}+C_{\text {mod }}^{-}
$$

where $C_{\text {res }}^{-}$is the residual coupling in the ring before the skew quadrupole modulation.

The modulation frequency is chosen small enough so that the tunes have enough time to equilibrate to respond to the coupling change. According to the simulation results and the preliminary beam experiments at RHIC, the skew quadrupole modulation frequency is chosen below $1.0 \mathrm{~Hz}$. Substituting Eqs. (5) and (6) into Eq. (4) gives

$$
\begin{aligned}
|\Delta Q|^{2}= & \Delta^{2}+\left|C_{\text {res }}^{-}\right|^{2}+\frac{1}{2}\left|C_{\text {mod, amp }}^{-}\right|^{2} \\
& +2\left|C_{\text {res }}^{-}\right|\left|C_{\text {mod,amp }}^{-}\right| \cos \Delta \chi \sin (2 \pi f t) \\
& -\frac{1}{2}\left|C_{\text {mod,amp }}^{-}\right|^{2} \cos (4 \pi f t),
\end{aligned}
$$

where $\Delta \chi$ is the angle difference between $C_{\text {res }}^{-}$and $C_{\text {mod,amp }}^{-}$

From Eq. (7), the $2 f$ term is only related to the skew quadrupole modulation amplitude $\left|C_{\text {mod,amp }}^{-}\right|$, while the $1 f$ term is related to the dot multiplication of the modulation coupling $C_{\text {mod }}^{-}$and the residual coupling $C_{\text {res }}^{-}$. So in the frequency domain of $|\Delta Q|^{2}$, there will be two peaks located at $1 f$ and $2 f$ if the machine is originally coupled, or only one peak at $2 f$ if the machine is originally well decoupled. The $1 f$ peak is, therefore, one of the reflections of the residual coupling.

\section{Projection ratio}

A straightforward use of Eq. (7) is the projection ratio of the residual coupling onto the direction of modulation coupling, which is defined as

$$
\kappa=\frac{\left|C_{\text {res }}^{-}\right| \cos \Delta \chi}{\left|C_{\text {mod,amp }}^{-}\right|},
$$

where $\left|C_{\text {res }}^{-}\right| \cos \Delta \chi$ is the residual coupling projection onto the coupling modulation direction. $\kappa$ is a dimensionless quantity. Its sign is determined by the difference angle $\Delta \chi$ between the residual and the modulation coupling.

The modulation coupling's $C_{\text {mod,amp }}^{-}$amplitude and direction can be calculated from the optics model according to Eq. (3). Knowing the projection ratio $\kappa$ and $\left|C_{\text {mod,amp }}^{-}\right|$, the coupling projection $\left|C_{\text {res }}^{-}\right| \cos \Delta \chi$ onto the modulation direction of $C_{\text {mod,amp }}^{-}$can be determined from Eq. (8).

In beam experiment, if the $1 f$ and $2 f$ peaks' amplitudes from the fast Fourier transformation (FFT) of $|\Delta Q|^{2}$ are $A_{1 f}, A_{2 f}$, then according to Eq. (8) and (7), the projection ratio is given by

$$
|\kappa|=\frac{A_{1 f}}{4 A_{2 f}} .
$$

The sign of the projection ratio is the same as the sign of $\sum_{j=1}^{N}|\Delta Q|_{t_{j}}^{2} i_{\text {mod }}\left(t_{j}\right)$, where $N$ is the number of the tunes $\left(Q_{1}, Q_{2}\right)$ given by the PLL system in one skew quadrupole modulation period. $i_{\text {mod }}$ is the skew quadrupole modulation current, $i_{\text {mod }}=I_{0} \sin (2 \pi f t)$, where $I_{0}$ is the modulation current's amplitude, $f$ is the skew quadrupole modulation frequency. Here we assume that skew quadrupole current change $I_{0}$ introduces $C_{\text {mod,amp }}^{-}$into the machine.

\section{Apply to RHIC coupling measurement}

Two projections onto two known coupling modulation directions are needed to fully determine the residual coupling. RHIC has three correction skew quadrupole families, $F 1, F 2$, and $F 3$. The coupling contributions to the total coupling from each skew quadrupole in one family are almost the same due to the sixfold lattice structure. The coupling contributions from these three skew quadrupole families with same strength amplitude and proper polarities are about $120^{\circ}$ angle apart. Knowing the projections onto at least two skew quadrupole family modulation directions, the residual coupling can be determined. Then the residual coupling correction can be carried out.

The above $120^{\circ}$ coupling angle difference is important to calculate the residual coupling values from the measured projection ratios. It can be proved that it holds for RHIC at injection, store and on the ramp, if the following conditions are met: (i) The betatron phase advances in each RHIC sextant are the same or almost the same. (ii) The integer split of the uncoupled tunes equals 1 . (iii) The fractional split of the uncoupled tunes is small.

It is wise to choose two orthogonal skew quadrupole modulations whose coupling contributions are $90^{\circ}$ apart. So if the same orthogonal skew quadrupole families are used for the measurement and the correction, the correction strengths can be directly obtained from the two projection 
ratios. Since RHIC has three skew quadrupole families, there are different combinations to construct these two orthogonal families. For example, $F 1$ and $F 3$ can be modulated simultaneously with the same frequency and same phase to produce a coupling normal to that only from the $F 2$ family modulation.

\section{DATA ACQUISITION AND ANALYSIS}

\section{A. PLL tune measurement}

The RHIC PLL system is used for fast tune measurement during the skew quadrupole modulation. Its data acquisition frequency is $177 \mathrm{~Hz}$, which is much faster than the skew quadrupole modulation frequency. Figure 1 shows the PLL tune data of three separate skew quadrupole modulations from the three families of the RHIC blue ring at injection. Figure 2 shows the detailed PLL tune data in the first skew quadrupole modulation in Fig. 1, where the skew quadrupole family $F 1$ was modulated. The skew quadrupole current modulation amplitude is $1.0 \mathrm{~A}$, the modulation frequency $1.0 \mathrm{~Hz}$.

\section{B. FFT}

The PLL tune data acquisition frequency is $177 \mathrm{~Hz}$, while the skew quadrupole modulation frequency is below $1.0 \mathrm{~Hz}$. To get better resolution of the FFT spectrum of $|\Delta Q|^{2}$ in the low frequency range, 2048 continuous sets of $Q_{1,2}$, or about $12 \mathrm{~s}$ of skew quadrupole modulation, are used for the FFT data processing. Figure 3 shows the FFT plot of the first modulation in Fig. 1. According to Eq. (9), the projection ratio of the residual coupling onto the $F 1$ family coupling contribution direction is 0.622 .

\section{Linear regression}

To further shorten the time for one residual coupling projection ratio measurement, data processing with linear regression [9] was used to calculate the projection ratio.

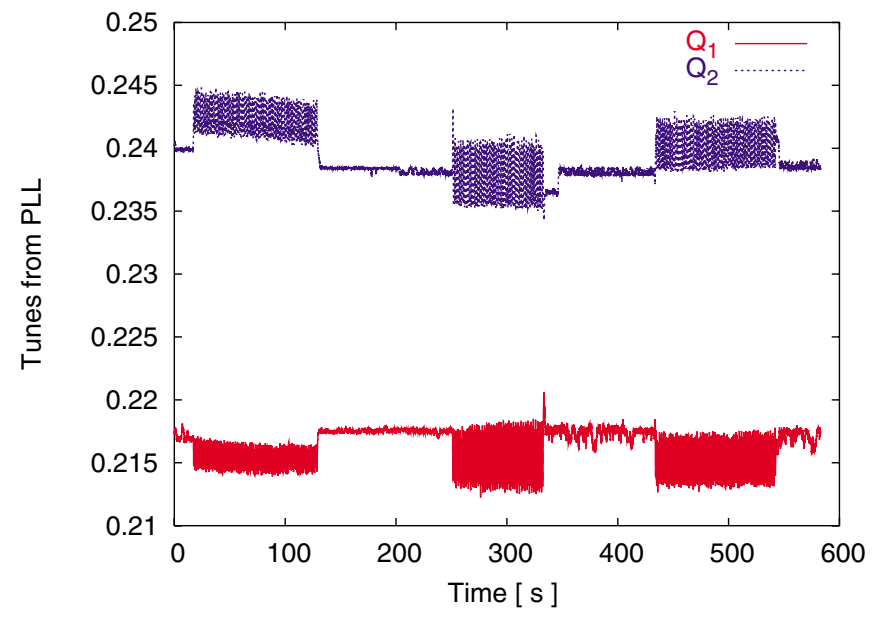

FIG. 1. (Color) PLL tune data of three modulations at injection.

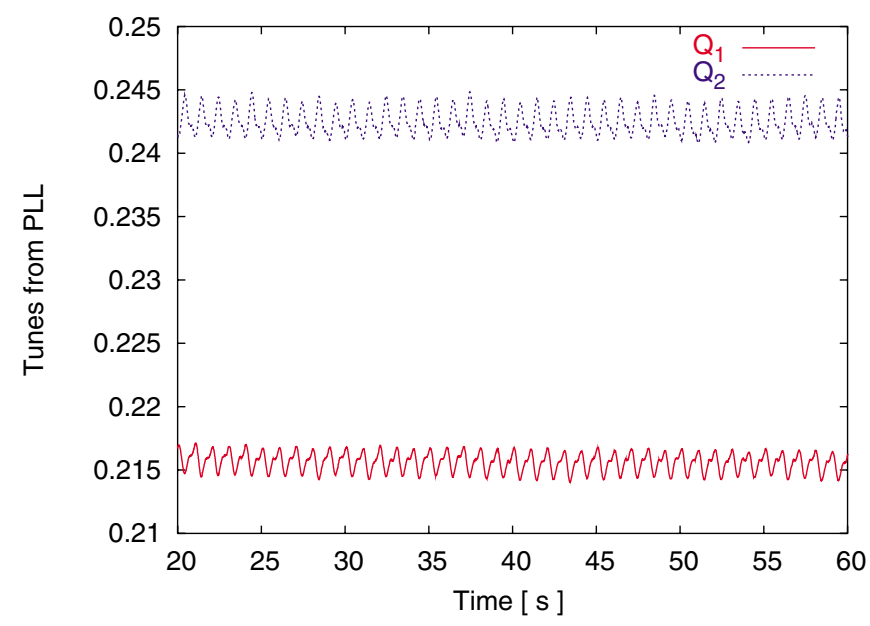

FIG. 2. (Color) Detailed tune data for the first modulation in Fig. 1.

According to Eq. (7), the fitting function was assumed to be

$$
\begin{aligned}
f\left(t_{i}\right)= & A+B_{1} \sin \left(2 \pi f t_{i}\right)+B_{2} \cos \left(2 \pi f t_{i}\right) \\
& +C_{1} \sin \left(4 \pi f t_{i}\right)+C_{2} \cos \left(4 \pi f t_{i}\right)+E t_{i}+F t_{i}^{2},
\end{aligned}
$$

where $A, B_{1}, B_{2}, C_{1}, C_{2}, E, F$ are the fitting coefficients. The linear and quadratic terms of time $E$ and $F$ are included here to overcome the possible tune shifts during modulation. Linear regression is used to minimize the error function

$$
\chi^{2}=\sum_{i=1}^{N}\left[|\Delta Q|_{i}^{2}-f\left(t_{i}\right)\right]^{2} .
$$

The projection ratio is, therefore, given by

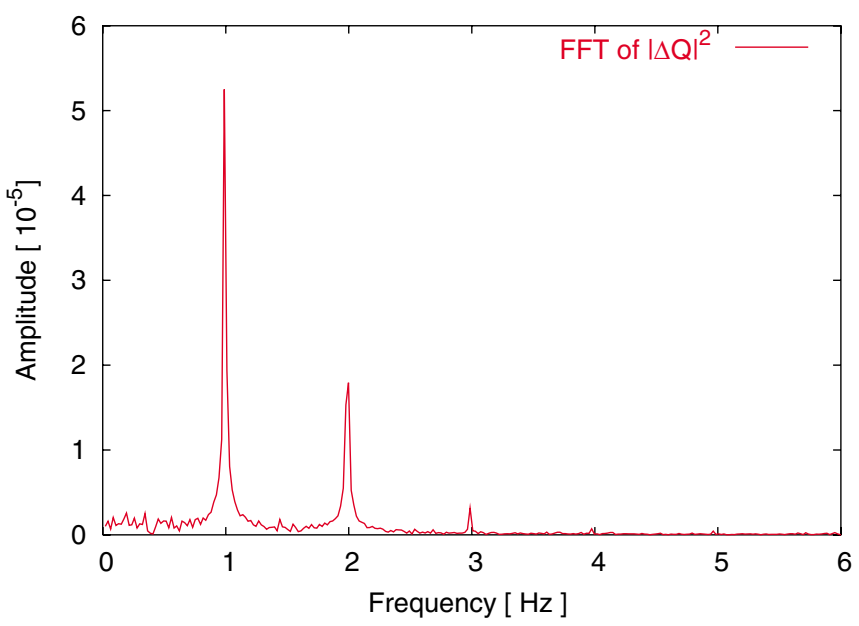

FIG. 3. (Color) FFT spectrum of $|\Delta Q|^{2}$ for Fig. 2 tune data. 


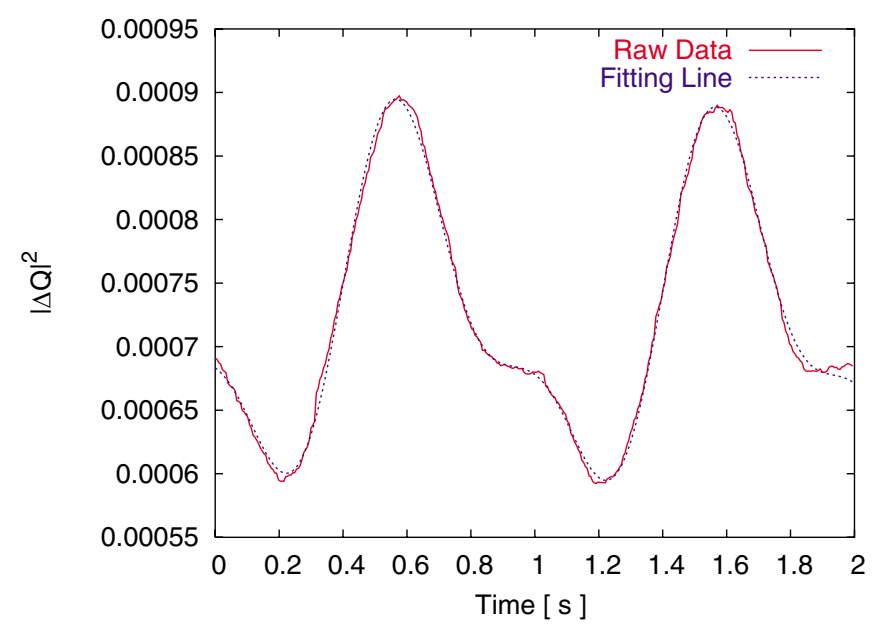

FIG. 4. (Color) Linear regression of $|\Delta Q|^{2}$ at injection.

$$
|\kappa|=\frac{\sqrt{B_{1}^{2}+B_{2}^{2}}}{4 \sqrt{C_{1}^{2}+C_{2}^{2}}} .
$$

For the linear regression data processing method, two or three skew quadrupole modulation periods are used to obtain the projection ratio.

Figure 4 shows one example of $|\Delta Q|^{2}$ fitting. The PLL tune data from Fig. 2 are used. Two modulation period tune split data are shown here. The projection ratio obtained from linear regression is 0.574 .

Figure 5 gives the continuous projection ratios obtained with the linear regression method. The tune data shown in Fig. 2 are used. The linear regression is performed every $2 \mathrm{~s}$, or two modulation periods.

Using linear regression technique, the time for one projection ratio measurement can be greatly reduced below $10 \mathrm{~s}$. However, FFT analysis is still needed sometimes,

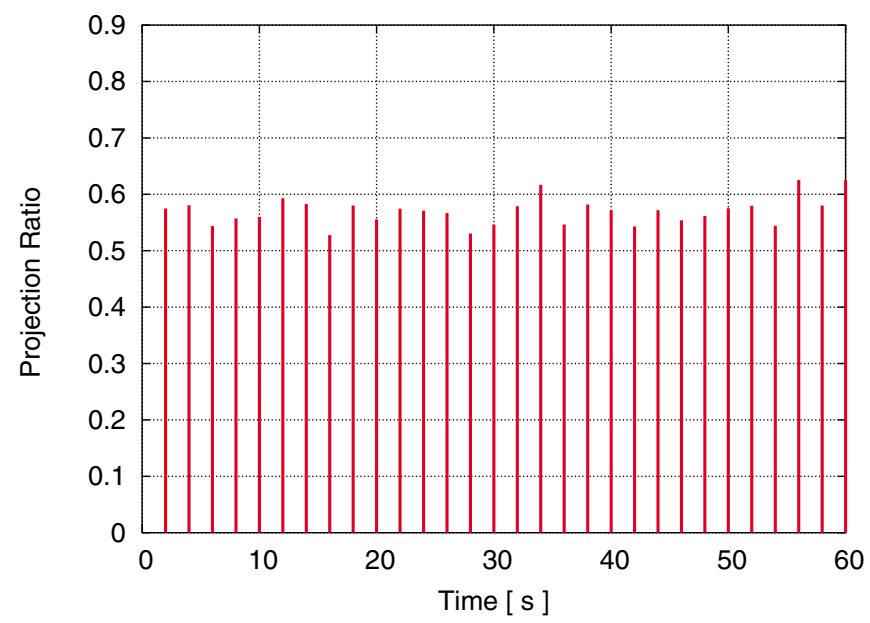

FIG. 5. (Color) Example of projection ratio continuous measurement. especially when the quality of the PLL tune data is not good enough. FFT data analysis is more robust and reveals more physics than does the linear regression analysis.

\section{BEAM EXPERIMENT RESULTS}

\section{A. Measurements at injection}

Table I shows one example of the residual coupling measurements at RHIC blue injection. The machine was coupled before the measurement. The three skew quadrupole families were modulated one by one to measure the residual coupling projection ratios. For each skew quadrupole family modulation, the skew quadrupole current modulation amplitude was $1.0 \mathrm{~A}$, and the modulation frequency was $1.0 \mathrm{~Hz}$. For the three projection ratio measurements, according to the RHIC optics model, their modulation coupling amplitudes $\left|C_{\text {mod,amp }}^{-}\right|$are the same since their current modulation amplitudes are the same.

Table I lists the measured residual coupling projection ratios and the projection directions. Table II gives the residual coupling calculated from either two of the above three projection measurements, where the residual coupling is given in the unit of the modulation coupling amplitude $\left|C_{\text {mod,amp }}^{-}\right|$. The average of the measured residual coupling projection ratio is 3.35 , the average of the measured residual coupling direction is about $338.7^{\circ}$.

\section{B. Measurements at store}

Table III gives one measurement example at RHIC store, where we modulated skew quadrupole families $F 1$ and $F 3$ simultaneously, producing a coupling modulation $(F 1 F 3)$ orthogonal to that only from family $F 2$ 's modulation. The top and bottom block of Table III give the measured projection ratios before and after family $F 3$ integrated strength change from -0.0004 to $-0.0002 \mathrm{~m}^{-1}$. For each skew quadrupole modulation, the skew quadrupole

TABLE I. One example of coupling measurements at injection.

\begin{tabular}{lcc}
\hline \hline Modulation family & Projection ratio & Projection direction $\left(^{\circ}\right)$ \\
\hline$F 1$ & 2.04 & $289.5^{\circ}$ \\
$F 2$ & 0.92 & $49.0^{\circ}$ \\
$F 3$ & 3.45 & $169.7^{\circ}$ \\
\hline \hline
\end{tabular}

TABLE II. Residual coupling from measurements.

\begin{tabular}{lcc}
\hline \hline Combination & $\begin{array}{c}\text { Residual coupling } \\
\text { projection ratio }\end{array}$ & $\begin{array}{c}\text { Residual coupling } \\
\text { direction }\left(^{\circ}\right)\end{array}$ \\
\hline$(F 1, F 2)$ & 3.00 & $336.8^{\circ}$ \\
$(F 2, F 3)$ & 3.59 & $333.8^{\circ}$ \\
$(F 3, F 1)$ & 3.47 & $343.6^{\circ}$ \\
\hline
\end{tabular}


TABLE III. One measurement at store.

\begin{tabular}{lcc}
\hline \hline Modulation & $\begin{array}{c}\text { Projection ratio } \\
\text { from FFT }\end{array}$ & $\begin{array}{c}\text { Projections ratio } \\
\text { from linear regression }\end{array}$ \\
\hline$F 2$ & 0.32 & 0.32 \\
$(F 1 F 3)$ & 0.22 & 0.19 \\
$F 2$ & 1.37 & 1.48 \\
$(F 1 F 3)$ & 2.53 & 2.45 \\
\hline \hline
\end{tabular}

current modulation amplitude was $1.0 \mathrm{~A}$, and the modulation frequency was $1.0 \mathrm{~Hz}$.

The integrated skew quadrupole strength change of $0.0002 \mathrm{~m}^{-1}$ should give $2.2 \mathrm{~A}$ change in the skew quadrupole power supplies' current. From the measurements, the averaged projection ratio changes averaged from FFT and linear regression onto $F 2$ and $(F 1 F 3)$ modulation directions are about 1.1 and 2.3 , respectively. The predications for the predication ratios are 1.1 and 1.9 , respectively.

\section{Measurements on the ramp}

Figure 6 shows one example of skew quadrupole modulation on the ramp, where the modulation frequency is $0.2 \mathrm{~Hz}$. The observed PLL tune losing lock in the end of modulation is possibly due to the very small tune split that the PLL system failed to distinguish. This small tune split may happen at the exact difference coupling resonance.

Figure 7 shows the projection ratios from the above measurement, where only the valid PLL tune data are used. The time scale is not the same as for Figs. 6 and 7. The linear regression is performed every two modulation periods, or $10 \mathrm{~s}$.

From Fig. 7, the projection ratio from the linear regression increases with time, which is due to the constant current amplitude modulation. In the future, the constant skew quadrupole strength amplitude modulation will replace the constant skew quadrupole current amplitude modulation on the ramp.

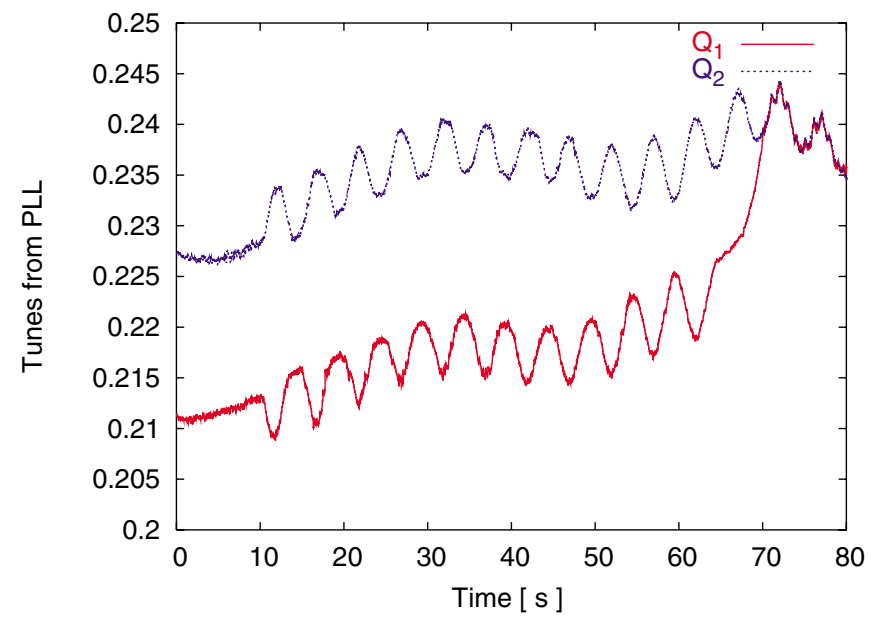

FIG. 6. (Color) An example of measurement on the ramp.

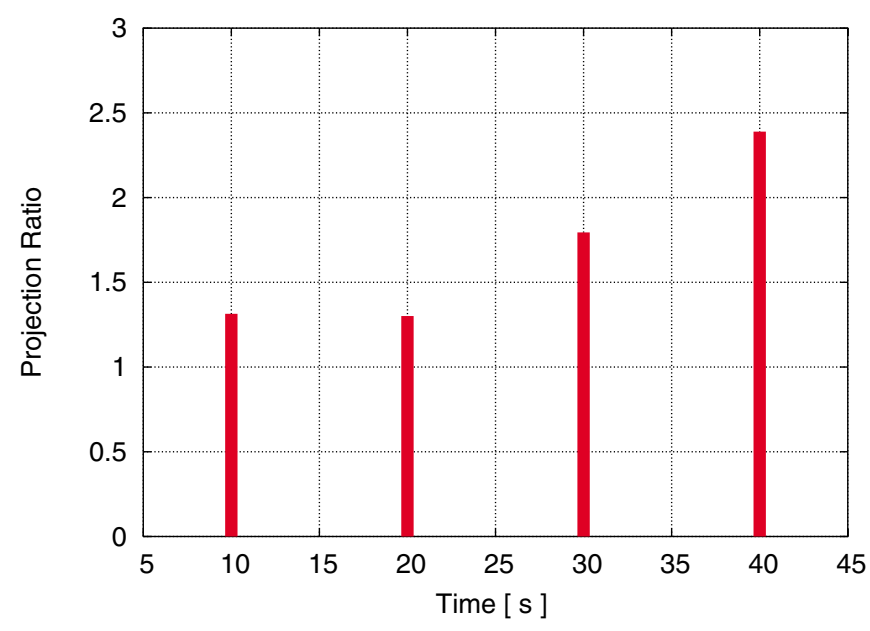

FIG. 7. (Color) Projection ratios on ramp with constant skew quadrupole current amplitude modulation.

To obtain good quality tunes from the PLL system was most important during the beam experiments on the ramp measurement. PLL losing lock was the big challenge in the skew quadrupole modulation measurement. To reduce the demands on the PLL system, the modulation time was shortened and linear regression data processing was used. Lowering the frequency of skew quadrupole modulation was found helpful on the ramp.

\section{CONCLUSION}

Skew quadrupole modulation is a promising technique to replace the skew quadrupole scan to measure the global coupling. This technique has several advantages that makes it suitable for coupling measurements and corrections on the ramp. The analytical solution to the tune split from the skew quadrupole modulation is given. Some preliminary results were obtained from the beam experiments in RHIC Run'04. The hardware requirements and challenges for this technique are also discussed. More beam experiments and online application are needed before the method is put into routine operation at RHIC.

\section{ACKNOWLEDGMENTS}

Y.L. thanks S. Peggs and R. Talman (Cornell) for the stimulating discussions on coupling observables. The operation group staffs are also thanked for their help during the beam experiments. This work is supported by the U.S. DOE under Contract No. DE-AC02-98CH10886.

[1] G. Guignard, CERN Report No. 76-06, 1976.

[2] G. Guignard, Phys. Rev. E 51, 6104 (1995).

[3] D. Rubin, in Handbook of Accelerator Physics and Engineering, edited by A.W. Chao and M. Tigner (World Scientific, Singapore, 1999). 
[4] F. Pila et al., "Coupling measurement and correction during RHIC run 2001 and development for 2003," 2002 (unpublished).

[5] W. Fischer, Phys. Rev. ST Accel. Beams 6, 062801 (2003).

[6] P. Cameron et al., in Proceedings of the 2004 European Particle Accelerator Conference, Lucerne, Switzerland, http://accelconf.web.cern.ch/AccelConf/, p. 524.
[7] P. Cameron et al., in Proceedings of the 2004 European Particle Accelerator Conference, Lucerne, Switzerland (Ref. [6]), p. 1294.

[8] H. Wiedemann, Particle Accelerator Physics II, Nonlinear and Higher-Order Beam Dynamics (Springer-Verlag, Berlin, 1995).

[9] S. C. Chapra and R. P. Canale, Numerical Methods For Engineers (McGraw-Hill, New York, 1988). 